\title{
MASKLESS 3D MICROFABRICATION OF DRUG-LADEN CAPSULATED MICROSTRUCTURES
}

\author{
Lin $Q i^{1}$, Shuai Yuan ${ }^{2}$, Ronald X. Xu ${ }^{1,2}$ and Yi Zhao ${ }^{{ }^{*}}$ \\ ${ }^{1}$ The Ohio State University, OH, USA \\ ${ }^{2}$ University of Science and Technology of China, Hefei, China
}

\begin{abstract}
This paper reports the development of 3D capsulated microstructures with the desired geometries for controlled drug releasing. Coaxial electrospray (CES) was used to encapsulate bioactive molecules/drugs in polymeric microcapsules. Microelectrodes were used to collect microcapsules and create particulate 3D microstructures. The shell thickness of the microcapsules and the characteristic dimensions of assembled microstructures were independently controllable, and both can modulate the drug releasing profiles. This approach can extend the releasing period and allow for programmable control of the drug-releasing profile. It thus provides a powerful tool for achieving customized drug releasing in the local regions.
\end{abstract}

\section{INTRODUCTION}

The microsphere-based scaffold is a promising scaffold candidate for promoting functional tissue regeneration [1]. The porosity of the scaffold mimics the native environment of cells, provides structural support and guidance of the regenerating tissue, regulates the interactions between the cells and their surroundings, and allows a good nutrient supply. The microspheres are the carriers of bioactive molecules or drugs, which can allow sustainable drug release at an optimized rate to promote tissue regeneration. Emulsion-solvent extraction is one conventional method to fabricate microspheres [2]. The scaffold materials and bioactive drugs are dissolved in the oil phase and emulsified as micro-droplets in the water phase. Upon the extraction of solvent from the oil phase, the droplets are hardened and solidified. Using this approach, microspheres are obtained at high efficiency and with simple settings. Nonetheless, emulsion-solvent extraction often has poor control over the microsphere size. Customized design of the drug-releasing profile is difficult. To address the broad size distribution, alternative approaches, such as precision particle fabrication and thermally induced phase separation, are developed $[3,4]$. However, these approaches have their particular limitations, such as complicated experimental setup and operation complexity, time consuming, and the risk of microsphere coalescence. Besides, bioactive drugs are often heterogeneously distributed in the microspheres, resulting in poor control of the initial drug releasing rate (e.g., initial burst release) and total releasing period [5].

CES is another prevalent approach to generate microspheres [6]. It can encapsulate bioactive molecules or drugs at a high efficiency [7] and have a relatively narrow size distribution as compared to emulsion-solvent extraction. The encapsulation and solvent evaporation often finish within seconds, which minimizes the adverse effect associated with the prolonged exposure of the solvent to bioactive molecules. The bioactive drugs are loaded in the cores of the microcapsules [8]. The initial burst release is thus minimized, and the releasing rate is tunable by controlling the shell thickness of microcapsules. However, there are few reports of functional tissue scaffolds created by CES. This is primarily due to the lack of feasible engineering approaches that can manufacture the microcapsules into desired scaffold shapes to support the tissue growth. So far, most microcapsules prepared by CES are discrete microparticles or particulate aggregates with random shapes. It is challenging to assemble the discrete microcapsules into $3 \mathrm{D}$ structures with controllable dimensions. Conventional microsphere assembling/packing methods, such as heat and solvent sintering, may nullify bioactive molecules in the microcapsules or leave cytotoxic residues in the assembled scaffolds, and thus compromise the efficacy of the functional microsphere-based scaffolds for tissue regeneration [1].

In this paper, we introduce a floating electrodes-assisted CES to assemble drug-laden microcapsules in a 3D microstructure during the collecting process. A coaxial flow with the inner drug solution and the outer polymer solution was electrosprayed in a stable cone-jet mode. Microcapsules were selectively deposited on the interdigitated microelectrodes due to the highly non-uniform electrostatic field generated by ground/floating electrodes. Continuous spraying stacked individual microcapsules into 3D capsulated microstructures. By tuning the voltage bias at the needle, the lateral dimension of these microstructures can be changed. The controlled drug releasing rate by such assembled capsulated microstructures was demonstrated. The results showed that both the shell thickness of microcapsules and the characteristic dimensions of the assembled microstructures can modulate the drug release profile. Our approach provides a new solution to control the drug-releasing profiles, validates and extends the potential efficacy of the CES. The study may shed light on the design of smart scaffolds for tissue regeneration.

\section{EXPERIMENTAL SECTION Materials}

Polycaprolactone (PCL, average $\mathrm{Mn}=45,000$, Sigma-Aldrich, USA) was dissolved in acetone (Sigma-Aldrich) in a concentration of $5 \%(\mathrm{w} / \mathrm{v})$ as the outer layer solution. To visualize the core-shell structure, the PCL solution was stained by $0.01 \%$ of coumarin 6 (Sigma-Aldrich) in green $(\mathrm{EX} / \mathrm{EM}=549 / 565 \mathrm{~nm})$, whereas the inner layer solution was stained by $0.01 \%$ of DiI (AnaSpec, Inc., USA) in red $($ excitation/emission $=528 / 617 \mathrm{~nm}) .70 \%$ isopropanol solution (IPA, Sigma-Aldrich) was used to wash and clean the electrodes before $3 \mathrm{D}$ patterning process. Deionized (DI) water was prepared by Direct-Q ${ }^{\circledR}$ Water Purification System (Millipore, Inc., USA). Rhodamine B (Sigma-Aldrich) was used as a model drug to demonstrate the release profiles of the resulting 3D patterning $(\mathrm{EX} / \mathrm{EM}=553 / 627 \mathrm{~nm})$.

\section{D Coaxial Electrospray Patterning}

The enhanced electrospray system consisted of a CES module, a collection module, and a monitor module [9]. To encapsulate drugs in the core of the microcapsules, a coaxial needle (Figure 1) with a $17 \mathrm{G} / 22 \mathrm{G}$ coaxial needle was used. The tips of the needles were flattened while the edges were rounded. The inner and the outer needles were positioned concentrically with a small vertical displacement $(0 \sim 0.3 \mathrm{~mm})$. The acetone solution and PCL solution were injected into the inner and outer layers of the needle respectively, using two syringe pumps (NE-1000, New Era Pump Systems, Inc., USA). A positive electrical voltage bias (from 8.5 to $12.5 \mathrm{kV}$ ) was applied to the needle (Gamma High Voltage Research, Inc., FL, USA). A negative voltage bias $(-10 \mathrm{kV})$ was applied to the 
collecting electrodes. The vertical distance between the needle tip and the collecting substrate was kept at $200 \mathrm{~mm}$. The interdigitated microelectrodes were fabricated using standard photolithography [10]. To identify the optimal conditions that can lead to high aspect ratio $3 \mathrm{D}$ assembled microstructures, a number of parameters were investigated: the flow rate $(1 \sim 5 \mathrm{ml} / \mathrm{h})$, the applied positive electric bias $(8.5 \sim 12.5 \mathrm{kV})$, the electrode width $(25 \sim 200 \mu \mathrm{m})$, the interelectrode spacing $(200 \sim 1000 \mu \mathrm{m})$ and the collecting time (0 50 $\mathrm{min})$. The entire process of electrospray was monitored in real time using a $3 \mathrm{kHz}$ strobe flashlight, a charge-coupled device camera (Allied vision technologies, Inc., MA), a computer and a microscopic lens (Model VT-7DS-2CD, Hangzhou, China).

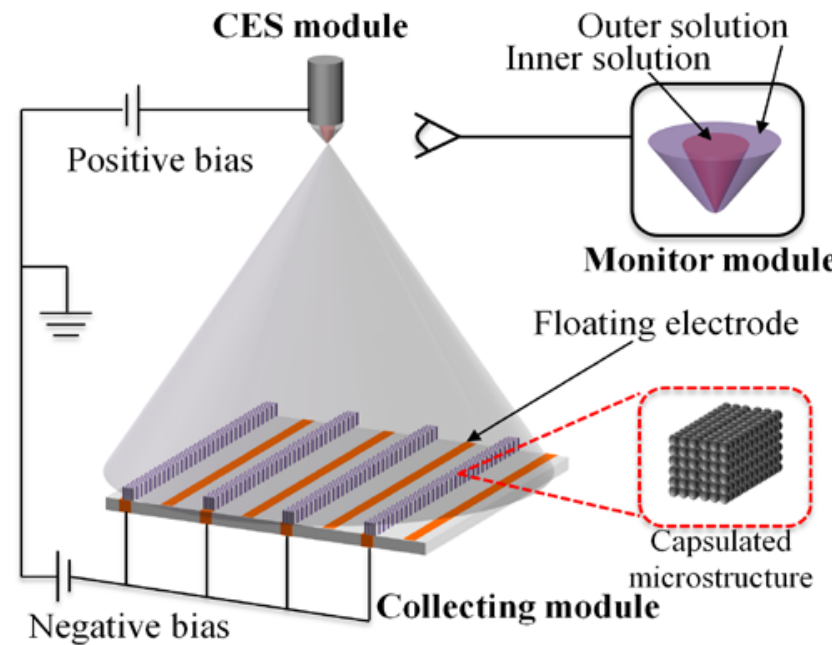

Figure 1: Schematic of the floating electrode assisted CES process. PCL solution and the acetone solution of the model drug (rhodamine B) were fed through the outer and inner layers of a coaxial needle $(17 G / 22 G)$.

\section{Morphology Characterization}

The morphology of the 3D microstructures was examined using scanning electron microscopy (SEM, A Hitachi S-3000H, Japan). In order to examine the core/shell configuration of the microcapsules, coumarin- 6 was added in the outer solution and DiI was added in the inner solution. The microcapsules were examined using fluorescence microscopy (ECLIPSE 80i, Nikon, Japan). The mean size and size distribution of microcapsules were determined from more than 200 particles using a bright field microscope (ECLIPSE LV100D, Nikon, Japan). The images were analyzed using the NIS-Elements AR software (Nikon Instruments Inc., USA).

\section{In Vitro Release Study}

The model drug release was determined by placing the assembled 3D microstructure and discrete microcapsules in $2 \mathrm{ml}$ phosphate-buffered saline (PBS). These samples were placed within the dialysis bags (Spectra/Por ${ }^{\circledR}$ Biotech Dialysis Membranes, Membranes package: 1M, MWCO 3.5K, W. $10 \mathrm{~mm}$, Diam. $6.4 \mathrm{~mm}$, Vol. $0.32 \mathrm{ml} / \mathrm{cm}$ ). The dialysis bags were placed at the bottom of $250 \mathrm{ml}$ glass beakers. The beakers were placed on a shaker (ROCKER 35, Labnet International Inc., USA) at $60 \mathrm{rpm}$ in a $37^{\circ} \mathrm{C}$ incubator. At the scheduled time intervals, the $3 \mathrm{ml}$ of the supernatant was collected, and replaced by the same amount of fresh PBS. The concentration of rhodamine B in the supernatant was determined by the light absorbance at $553 \mathrm{~nm}$ using a UV-Visible spectrophotometer (50 Bio, Agilent Technologies, USA). To establish the standard releasing rate, pure rhodamine B of the same dose was measured. The experiment was performed in dark. Each experiment was conducted in triplicate.

\section{RESULT AND DISCUSSION \\ Investigation of CES Instability}

The instability of CES was studied by examining the shape of the Taylor cone at the needle tip and resulting core/shell microcapsules. The results showed that the instability of the coaxial flow depends on the total flow rate and the electrostatic field. A high total flow rate or a low voltage bias resulted in the dripping mode of electrospray (I in Figure 2a), whereas a low total flow rate or a high voltage bias resulted in the multi-jet mode (III in Figure 2a). These modes may cause a broad size distribution and a significant variation of the drug-laden dose. When the total flow rate and the applied voltage bias were in the optimized range (II in Figure 2a), the CES system was operated with a stable cone-jet state, where a Taylor cone formed at the tip of the needle, and a liquid jet was ejected from the vertex of the cone. The jet then fissured into droplets with drug-containing cores. The shell dried during the flying process (Figure 2c). When the microcapsules approached the collecting substrate, they were attracted towards the electrodes with the negative voltage bias due to the non-uniform electric field. Since the microelectrodes collected most of the capsules and very few microcapsules fall into other regions, the waste of drugs was minimized. The geometries of the assembled pattern can be designed by changing the electrode layout (Figure $2 b \& d$ ). As the spray proceeded, the microcapsules stacked up and eventually formed the $3 \mathrm{D}$ microstructures with particulate morphology.
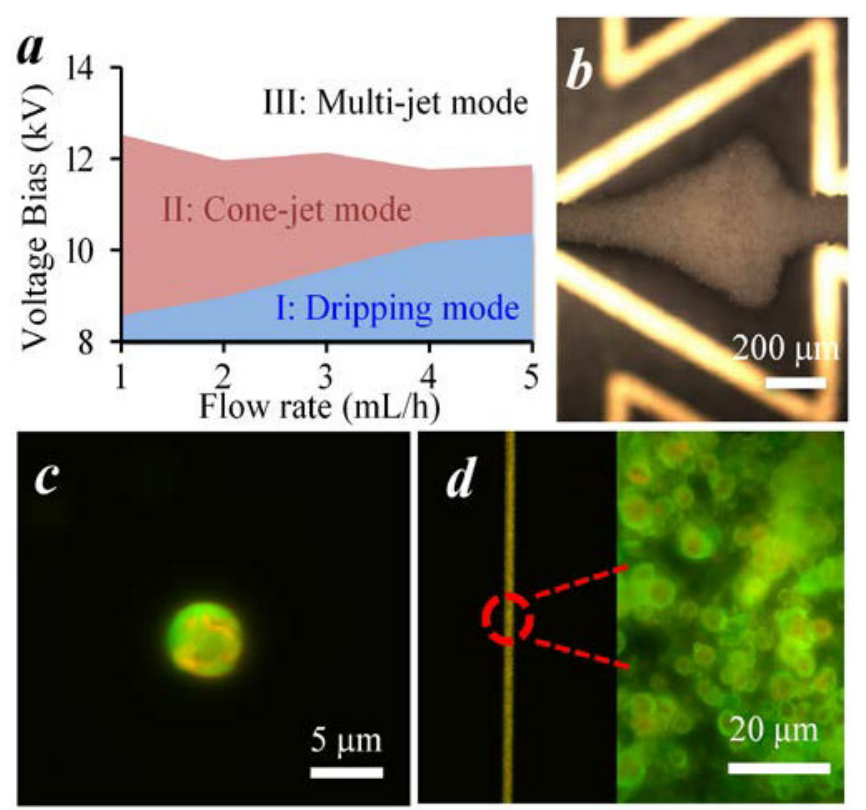

Figure 2: Formation of the capsulated microstructure assemblies. (a) The effect of the total flow rate and the voltage bias on the CES instability. Region I: dripping mode; Region II: cone-jet mode; Region III: multi-jet mode. (b) A microstructure defined by a triangle ground/floating electrode layout. (c) An individual microcapsule. (d) An assembled capsulated microstructure. The cores of the microcapsules were stained by DiI (red) and shells were stained by coumarin 6 (green).

\section{The Effect of the Operating Parameters}

The effects of the operating parameters on the characteristic dimensions of assembled microstructures were investigated. The results showed that the height-to-width ratio of these 
microstructures varied with the electrode layout, the spraying time and the applied voltage bias. For example, when the voltage bias and the spraying time were constant $(20 \mathrm{kV}$ and $30 \mathrm{~min})$, the electrode width of $50 \mu \mathrm{m}$ and the inter-electrode space of $400 \mu \mathrm{m}$ can lead to the highest height-to-width aspect ratio (about 3.02) (Table $1)$.

Table 1 The effect of the width and the interelectrode spacing on the height-to-width aspect ratios of the assembled microstructures.

\begin{tabular}{|c|c|c|c|c|}
\hline \multirow{2}{*}{$\begin{array}{c}\text { Height-to-weight Aspect } \\
\text { Ratios }\end{array}$} & \multicolumn{3}{|c|}{ Electrode Width $(\boldsymbol{\mu m})$} \\
\cline { 3 - 5 } & 25 & 50 & 100 \\
\hline \multirow{3}{*}{$\begin{array}{c}\text { Inter-electrode } \\
\text { spacing }(\boldsymbol{\mu m})\end{array}$} & 200 & 1.1 & 2.13 & 1.64 \\
\cline { 2 - 5 } & 100 & 1.56 & $\mathbf{3 . 0 2}$ & 1.85 \\
\cline { 2 - 5 } & 1000 & 1.72 & 1.82 & 1.24 \\
\hline
\end{tabular}

When the electrode layout and the voltage bias were kept unchanged, the height-to-width ratio of the assemblies increased with the spraying time at the beginning of the spraying. Almost all microcapsules were collected on the collecting electrodes with negative voltage bias (Figure 2d). The assembled microstructures thus had a good patterning contrast (Figure $4 a$ ). With continuous spraying, the height of the microstructure increased, whereas the width did not increase as much. Capsulated microstructures with higher aspect ratio were thus obtained (Figure $4 b$ ). As the spraying proceeded, the collecting electrodes were covered by a thick layer of the dielectric microcapsules. The non-uniformity of the electrostatic field reduced [10]. As a result, some microcapsules started to be deposited outside the collecting electrodes. This increased the lateral dimensions of the capsulated microstructures. The aspect ratio of the assembled structures decreased accordingly. In this study, the maximal height-to-width ratio was observed under the voltage bias of $20 \mathrm{kV}(+10 \mathrm{kV}$ at the needle and $-10 \mathrm{kV}$ at the collecting electrodes) and 40 min of spraying (Figure 3 ).

More complicated capsulated structures with varied lateral dimensions can be obtained by dynamically tuning the voltage bias at the needle. As shown in Figure $4 c \& d$, a capsulated microstructure with two segments with distinct widths was obtained. This microstructure was created by setting the voltage biases at the needles at $+8.5 \mathrm{kV}$ for $20 \mathrm{~min}$, and then changing the voltage to $+12.5 \mathrm{kV}$ and holding the voltage for another $20 \mathrm{~min}$.

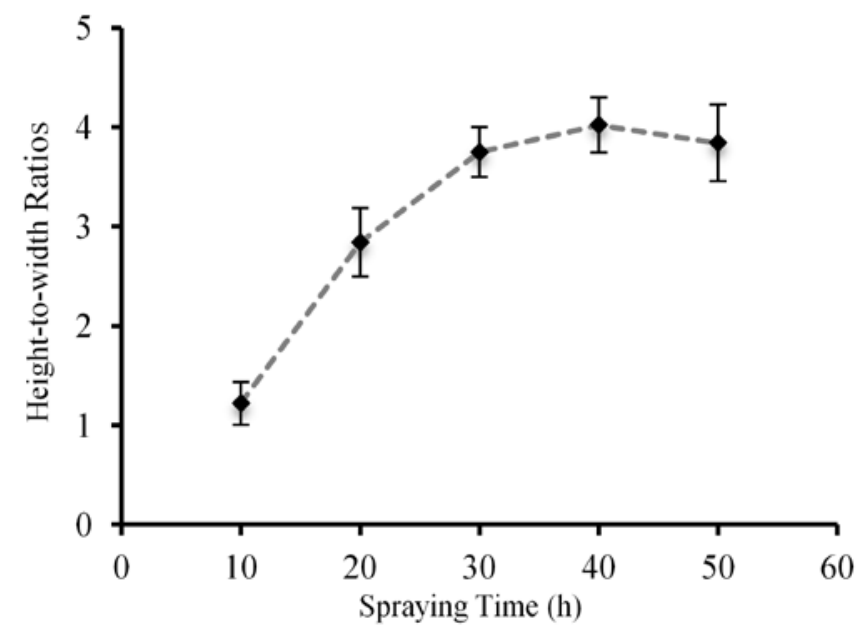

Figure 3: The effect of microcapsule spraying time on the height-to-width ratio of the capsulated microstructures.
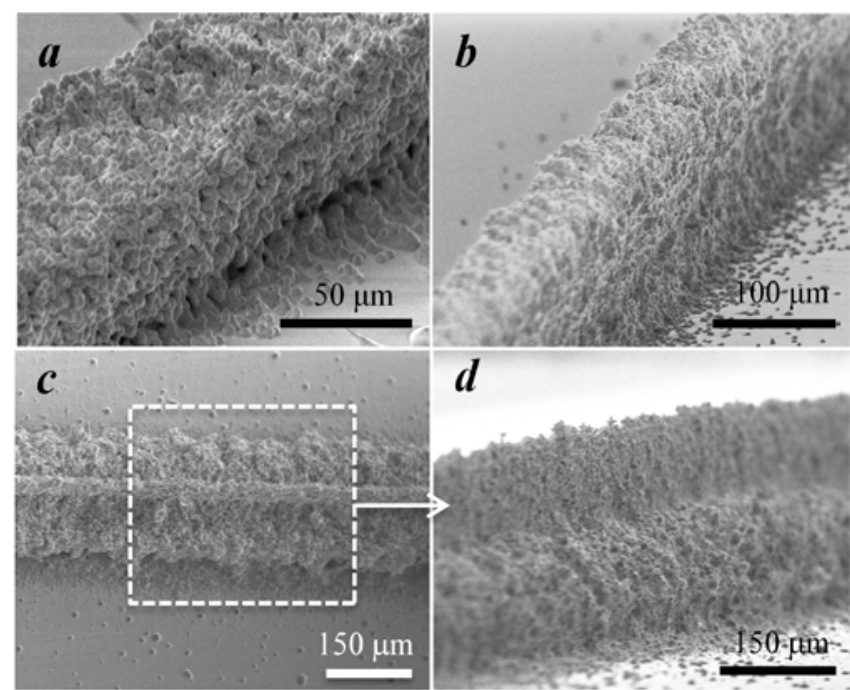

Figure 4: The SEM of the capsulated microstructures. (a-b) The capsulated microstructure assemblies with different height-to-width ratios. (c-d) A capsulated microstructure assembly with two segments with distinct cross-sectional dimensions.

\section{Effect of Geometry of Individual Microcapsules on the Releasing Rate}

Microcapsules with varied shell thicknesses were fabricated by using the different flow rates of the outer solution, and the same flow rate of the inner solution. The resulting capsules thus have the same core size but different shell thicknesses. Using CES, these microcapsules were assembled into capsulated microstructures with $200 \mu \mathrm{m} \times 200 \mu \mathrm{m} \times 20 \mathrm{~mm}$ (Figure 5a). The in vitro drug release experiment showed that the releases of all capsulated microstructures were sustainable up to one week (Figure $5 b$ ). The microstructure comprising of microcapsules with a thinner shell exhibited a faster-releasing rate the first 12 hours of the immersion. This suggested that the release of bioactive molecules or drugs from the microcapsulated scaffold can be modulated by changing the geometry of individual microcapsules.

\section{Effect of the Shape of Capsulated Microstructures on the Releasing Rate}

To investigate the effects of shape of the assembled capsulated microstructures on the releasing profile, microcapsules with the same geometries were assembled into microstructures. The cross-sectional dimensions of these microstructures were $50 \mu \mathrm{m} \times 50 \mu \mathrm{m}$ and $200 \mu \mathrm{m} \times 200 \mu \mathrm{m}$ (Figure 6a). The longitudinal lengths of both microstructures were $\sim 20 \mathrm{~mm}$. The discrete microcapsules were also tested as the control. The in vitro drug release experiment showed that both capsulated microstructures exhibited extended releasing profiles than the discrete microcapsules (Figure 6b). The capsulated microstructures with the greater cross-sectional dimensions exhibited a significantly lower releasing rate within first 48 hours of the immersion.

In previous drug release studies using microcapsules, the drug release profile is often modulated only by the core-shell geometry of microcapsules. A thick shell with a small core was often used to extend the releasing period and to minimize the initial burst. This, however, limits the total dose of the drug that can be carried by the microcapsules. Our approach provides an additional route to control the drug releasing profile by changing the shape of the capsulated microstructures. Extended releasing profile can be obtained without reducing the total dose of the drug by stacking the microcapsules with appropriate core/shell geometries into the microstructure 
assemblies with desired shape. The characteristic dimensions of the microstructures can be varied within a wide range from a few to hundreds of micrometers, which adds more flexibility for designing customized release profiles. Moreover, when the microcapsules carrying different drugs were used to construct the different segments of the capsulated structures that have different cross-sectional dimensions (as those shown in Figure 4c\&d), the releasing of multiple drugs with distinct releasing profiles can be implemented.

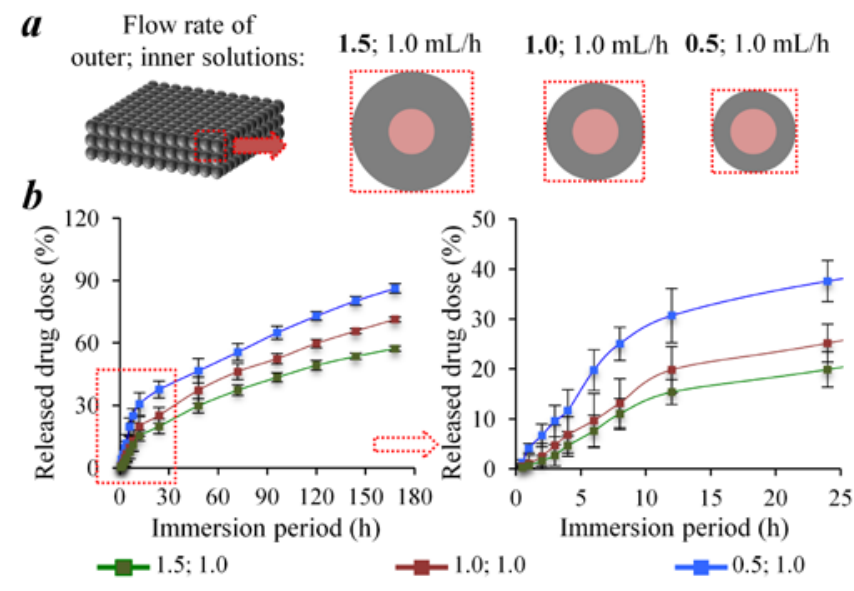

Figure 5: The effect of the shell thickness of individual microcapsules on the release profile. (a) Microspheres with varied shell thickness were generated by modulating the flow rate of the outer fluid; and (b) The drug-releasing profile over 175 hours of immersion.

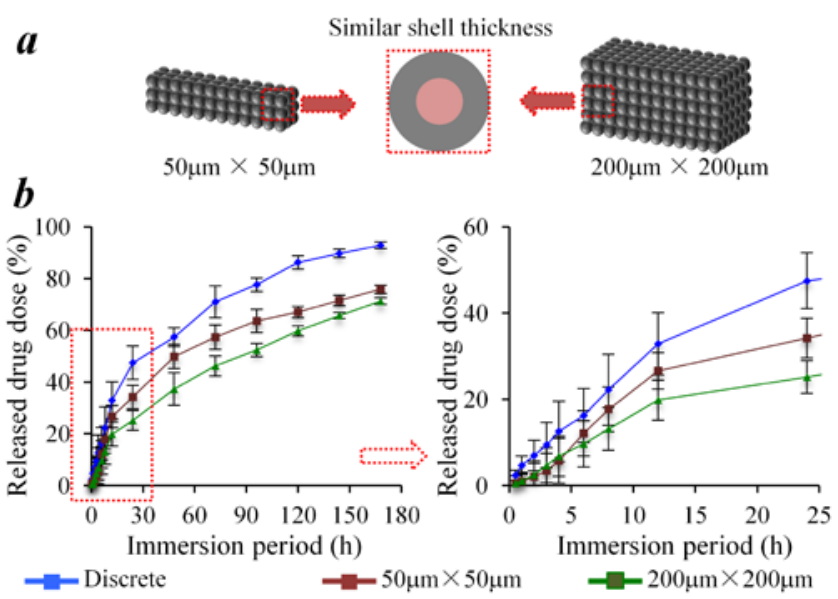

Figure 6: The drug release profile changes with the shape of the microstructure assemblies. Two capsulated microstructure assemblies with the cross-sectional area of $50 \mu \mathrm{m} \times 50 \mu \mathrm{m}$ and 200 $\mu \mathrm{m} \times 200 \mu \mathrm{m}$ (height $\times$ width) were tested. The lengths of the microstructure assemblies were $20 \mathrm{~mm}$.

\section{CONCLUSION}

In this paper, we report an approach to create 3D capsulated microstructure assemblies with desired geometries for controlled drug release. The floating electrode assisted coaxial electrospray was used to create microstructures comprising microcapsules that encapsulate bioactive molecules/drugs. The shell thickness of the microcapsules and the shape of the assembled microstructures are independently controllable, and both can modulate the drug releasing profile. This approach provides a powerful tool for obtaining the desired drug releasing profile in the local area that is otherwise difficult to obtain.

\section{REFERENCES}

[1] V. Gupta, Y. Khan, C. J. Berkland, C. T. Laurencin, and M. S. Detamore, "Microsphere-Based Scaffolds in Regenerative Engineering," Annu Rev Biomed Eng, vol. 19, pp. 135-161, Jun 212017.

[2] C. T. Laurencin, F. K. Ko, M. A. Attawia, and M. D. Borden, "Studies on the development of a tissue engineered matrix for bone regeneration," Cells and materials, vol. 8, pp. 175-181, 1998.

[3] J. J. Blaker, J. C. Knowles, and R. M. Day, "Novel fabrication techniques to produce microspheres by thermally induced phase separation for tissue engineering and drug delivery," Acta Biomater, vol. 4, pp. 264-72, Mar 2008.

[4] C. Berkland, K. Kim, and D. W. Pack, "Fabrication of PLG microspheres with precisely controlled and monodisperse size distributions," J Control Release, vol. 73, pp. 59-74, May 18 2001.

[5] Y. Yeo and K. Park, "Control of encapsulation efficiency and initial burst in polymeric microparticle systems," Arch Pharm Res, vol. 27, pp. 1-12, Jan 2004.

[6] L. de Juan and J. F. de la Mora, "Charge and Size Distributions of Electrospray Drops," J Colloid Interface Sci, vol. 186, pp. 280-93, Feb 151997.

[7] L. Zhang, J. Huang, T. Si, and R. X. Xu, "Coaxial electrospray of microparticles and nanoparticles for biomedical applications," Expert Rev Med Devices, vol. 9, pp. 595-612, Nov 2012.

[8] Y. H. Lee, F. Mei, M. Y. Bai, S. Zhao, and D. R. Chen, "Release profile characteristics of biodegradable-polymer-coated drug particles fabricated by dual-capillary electrospray," J Control Release, vol. 145, pp. 58-65, Jul 12010.

[9] S. Yuan, F. Lei, Z. Liu, Q. Tong, T. Si, and R. X. Xu, "Coaxial Electrospray of Curcumin-Loaded Microparticles for Sustained Drug Release," PLoS One, vol. 10, p. e0132609, 2015.

[10] X. Zhang and Y. Zhao, "Programmable patterning of polymeric microparticles by floating electrodes-assisted electrospray," Journal of Micromechanics and Microengineering, vol. 22, Apr 2012.

[11] M. Krampera, G. Pizzolo, G. Aprili, and M. Franchini, "Mesenchymal stem cells for bone, cartilage, tendon and skeletal muscle repair," Bone, vol. 39, pp. 678-83, Oct 2006.

[12] J. P. Vacanti and R. Langer, "Tissue engineering: the design and fabrication of living replacement devices for surgical reconstruction and transplantation," Lancet, vol. 354 Suppl 1, pp. SI32-4, Jul 1999.

[13] N. Mohan, V. Gupta, B. Sridharan, A. Sutherland, and M. S. Detamore, "The Potential of Encapsulating "Raw Materials" in 3D Osteochondral Gradient Scaffolds," Biotechnology and Bioengineering, vol. 111, pp. 829-841, Apr 2014.

[14] V. Gupta, N. Mohan, C. J. Berkland, and M. S. Detamore, "Microsphere-Based Scaffolds Carrying Opposing Gradients of Chondroitin Sulfate and Tricalcium Phosphate," Front Bioeng Biotechnol, vol. 3, p. 96, 2015.

\section{CONTACT}

*Yi Zhao, zhao.178@,osu.edu; Tel: 614-247-7424 